\title{
Enhanced Monthly Precipitation Forecasting Using Artificial Neural Network and Singular Spectrum Analysis Conjunction Models
}

\author{
Aman Mohammad Kalteh ${ }^{1}$
}

Received: 31 March 2017 / Accepted: 16 June 2017 / Published online: 27 June 2017

(C) Indian National Academy of Engineering 2017

\begin{abstract}
Precipitation is a complex atmospheric process with both temporal and spatial variations which make it difficult to forecast. Several models have been developed to forecast precipitation. This study investigates the accuracy of artificial neural network (ANN) and singular spectrum analysis (SSA) conjunction model in monthly precipitation forecasting. Here, SSA is used to preprocess raw input signals to provide data of high quality to ANN. The conjunction model is obtained by combining two methods, ANN and SSA, and compared with the single/regular ANN. In SSA, the window length and the optimum number of components must be determined. For this purpose, several tentative models, one for each of the available component numbers, are developed, and the related component number of the model with best performance is considered as the optimum number of components. If the selected optimum number is less or equal to the chosen window length which in turn indicates the adequacy of the chosen window length. Monthly precipitation data from one rain gauge station, Ponel station, in northern Iran, is used in the study. The root mean square error (RMSE), correlation coefficient $(\mathrm{R})$ and coefficient of efficiency (CE) statistics are used as the comparing criteria. The comparison of the results reveals that the conjunction models could increase the forecast accuracy of the ANN model in monthly precipitation forecasting. It is found that the conjunction model with $R M S E=52.257, R=0.858$ and $C E=0.731$ in the validation/testing period is superior
\end{abstract}

Aman Mohammad Kalteh

kalteh@guilan.ac.ir

1 Department of Range and Watershed Management, Faculty of Natural Resources, University of Guilan, P.O. Box 1144, Sowmehe Sara, Guilan Province, Iran in forecasting monthly precipitations than the most accurate ANN model with $R M S E=91.096, R=0.444$ and $C E=0.183$, respectively.

Keywords Precipitation - Data decomposition - Artificial neural networks $\cdot$ Singular spectrum analysis

\section{Introduction}

Rainfall/precipitation is one of the most important and complex components of the hydrological cycle, which exhibits both spatial and temporal variations due to complex mechanisms involved in generating of this atmospheric process. Hence, rainfall forecasting is known as a difficult task among hydrologists/water resources engineers community who deal with the modelling of this process. Nevertheless, for water resources planning purposes, a long-term rainfall series is needed in many hydrological and simulation models (Tantanee et al. 2005; Venkata Ramana et al. 2013). There have been many attempts to address the hydrological processes in general and the quantitative precipitation forecasting in particular through different techniques including numerical weather prediction models and remote sensing observations (Yates et al. 2000; Ganguly and Bras 2003; Diomede et al. 2008; He et al. 2013), statistical models (Chu and He 1994; Chan and Shi 1999; DelSole and Shukla 2002; Munot and Kumar 2007; Li and Zeng 2008; Nayagam et al. 2008), and soft computing methods including artificial neural network (ANN), self-organizing map (SOM), support vector machine (SVM), fuzzy inference system and extreme learning machine (ELM) (French et al. 1992; Navone and Ceccatto 1994; Pongracz et al. 2001; Freiwan and Cigizoglu 2005; Marzano et al. 2006; Kalteh and Berndtsson 
2007; El-Shafie et al. 2011; Chen et al. 2015; Gholami et al. 2015; Taormina and Chau 2015). For example, an ANN model for forecasting rainfall intensity fields at a lead-time of $1 \mathrm{~h}$ was applied by French et al. (1992). Pongracz et al. (2001) used fuzzy inference for monthly rainfall forecasting. Marzano et al. (2006) compared the accuracy of ANN approach with the previously developed regression methods in estimating precipitation intensity. El-Shafie et al. (2011) developed adaptive neuro-fuzzy inference system and ANN models for rainfall forecasting in Klang River, Malaysia. Taormina and Chau (2015) employed single and multi-objective binary-coded discrete fully informed particle swarm optimizations (BFIPS) and ELM to develop fast and accurate input variable selection algorithms for rainfall-runoff modelling.

A hydrological time series (e.g., rainfall time series) can be actually considered as a combination of stochastic (random)/noise and deterministic components (Salas et al. 1985). It is believed that once the noise component of the hydrologic series is appropriately removed using a suitable data-preprocessing technique, the deterministic component can then be easily modeled (Wu et al. 2010). There have been many attempts to address this issue in hydrological and atmospheric data through different techniques (Lisi et al. 1995; Sivapragasam et al. 2001; Chau and Wu 2010; Wu et al. 2010; Wu and Chau 2011; Kalteh 2013, 2015; Wang et al. 2015; Kalteh 2016). For example, Lisi et al. (1995) used SSA to extract significant components from the southern oscillation index and applied ANN for forecasting. Wu et al. (2010) developed modular ANNs coupled with data-preprocessing techniques including moving average (MA), principal component analysis (PCA) and SSA for the rainfall time series prediction. The performance of their prediction models improved once these models coupled with data-preprocessing techniques. Their study also indicated that the improvement of model performance generated by SSA is considerable compared to those of MA and PCA. They also argue that the essence of SSA in improving model performance is to strengthen the mapping relation of model input and output by removing noises in the original time series. Recently, Wang et al. (2015) presented the auto-regressive integrated moving average (ARIMA) model coupled with the ensemble empirical mode decomposition (EEMD) for forecasting annual runoff time series. More recently, Kalteh (2016) developed least squares support vector machine (LSSVM) models coupled with discrete wavelet analysis and SSA for forecasting monthly streamflow. The above studies indicate the importance of applying an appropriate data-preprocessing technique (e.g., SSA) to raw input signals to provide data of high quality to forecasting models (e.g., artificial intelligence models such as ANN) prior to introducing them to the models as input. Therefore, the main contributions of this study are as follows:

1. Development of SSA-based ANN models for monthly precipitation forecasting for a rain gauge station in northern Iran using various combinations of precipitation data of three previous months as input.

2. Determining the window length and the optimum number of components in SSA.

3. Comparison of ANN and SSA conjunction models with regular ANN models.

The rest of this paper is organized as follows. Firstly, we briefly describe ANN, SSA as well as study area and data. Then, results of this study are elaborated. Finally, some conclusions are made.

\section{Materials and Methods}

\section{Artificial Neural Networks (ANNs)}

An artificial neural network (ANN) is a non-linear black box statistical approach with a flexible mathematical structure that is capable of modelling non-linear relationships between input and output data without the necessity of detailed understanding of the complex physical laws governing the process under investigation. Over the past 20 years, ANNs have been used increasingly for hydrological forecasting. It is a massively parallel-distributed information processing system resembling biological neural networks of the human brain (American Society of Civil Engineers Task Committee 2000). The most commonly used ANN structure is the feed-forward multilayer perceptron. This structure is composed of three layers: an input layer, one or more hidden layers, and an output layer. The network topology consists of a set of neurons connected by links and normally organized in a number of layers. The number of neurons in the input and output layers is equal to the number of input and output variables, respectively. The number of neurons in the hidden layer (s) is usually selected by a trial-and-error procedure.

There are many types of algorithms available for training multilayer perceptron. In this study, multilayer perceptrons were trained using the Levenberg-Marquardt algorithm. This algorithm was developed to approach second-order training speed without having the computation of Hessian matrix (Demuth and Beale 1998). The Levenberg-Marquardt algorithm is more powerful than the conventional gradient descent techniques (Hagan and Menhaj 1994) although it requires a greater amount of memory (Adamowski and Sun 2010). 


\section{Singular Spectrum Analysis (SSA)}

The singular spectrum analysis (SSA) technique is a novel and powerful technique of time series analysis that has recently found many hydrological applications. According to Golyandina et al. (2001), the SSA technique involves two complementary stages: decomposition and reconstruction. Consider a real-valued time series $F=$ $\left\{x_{1}, \ldots, x_{N}\right\}$ of length $N$. Let $L$ be some integer (window length), $1 \prec L \prec N$, to be determined by the user. Here, these two stages are briefly presented and more details can be found in several publications (Golyandina et al. 2001; Rocco 2013; Golyandina and Korobeynikov 2014).

\section{1st Stage: Decomposition}

The decomposition stage consists of two steps: embedding and singular value decomposition (SVD).

\section{Embedding}

The embedding procedure maps the original time series into a sequence of lagged vectors of size $L$ by forming $K=N-L+1$ lagged vectors $X_{i}=\left\{x_{i}, \ldots, x_{i+L-1}\right\}^{T}$, $i=1, \ldots, K$. The trajectory matrix of the time series $F$ is

$X=\left[X_{1}, \ldots, X_{K}\right]=\left(\begin{array}{ccccc}x_{1} & x_{2} & x_{3} & \cdots & x_{K} \\ x_{2} & x_{3} & x_{4} & \cdots & x_{K+1} \\ x_{3} & x_{4} & x_{5} & \cdots & x_{K+2} \\ \vdots & \vdots & \vdots & \ddots & \vdots \\ x_{L} & x_{L+1} & x_{L+2} & \cdots & x_{N}\end{array}\right)$

The trajectory matrix is a Hankel matrix because $X$ has equal elements on anti-diagonals.

\section{$S V D$}

From matrix $X$, define the covariance matrix $X X^{T}$. The SVD of $X X^{T}$ provides a set of $L$ eigenvalues in the decreasing order of magnitude $\left(\lambda_{1} \geq \lambda_{2} \geq \cdots \geq \lambda_{L} \geq 0\right)$ and the corresponding eigenvectors $U_{1}, U_{2}, \ldots, U_{L}$. Then the SVD of the trajectory matrix $X$ can be written as

$X=X_{1}+\cdots+X_{L}$

where $X_{i}=\sqrt{\lambda_{i}} U_{i} V_{i}^{T}$. The matrices $X_{i}$ are elementary, that is, they have rank $1 . V_{i}=X_{i}^{T} U_{i} / \sqrt{\lambda_{i}}, \quad i=1, \ldots, L$, (equivalent to the $i$ th factor vector of $X X^{T}$ ). The triple $\left(\sqrt{\lambda_{i}}, U_{i}, V_{i}\right)$ is referred to as the $i$ th eigentriple.

\section{2nd Stage: Reconstruction}

This stage is subdivided into two steps: grouping and diagonal averaging.

\section{Grouping}

The grouping procedure partitions the set of indices $\{1, \ldots, L\}$ into $r$ (to be selected by the user) disjoint subsets. Let $I=\{i 1, i 2, \ldots, i r\}$ be a group of $r$ selected eigentriples and $X_{I}=X_{i 1}+X_{i 2}+\cdots+X_{i r}$, where $X_{I}$ is related to the signal of $F$ while the remaining $(L-r)$ eigentriples denotes the time series related to the error term.

\section{Diagonal Averaging}

Once the group of $r$ components selected, the next step is to reconstruct the deterministic components of the time series. The main principle to follow is to transform each of the terms $X_{i 1}, X_{i 2}, \ldots, X_{i r}$ into reconstructed time series $F_{i 1}, F_{i 2}, \ldots, F_{i r}$ via the Hankelization process $H()$ or diagonal averaging: assuming $b_{i j}$ as an element of a generic matrix $B$, the sth term of the reconstructed time series could be obtained by averaging $b_{i j}$, if $i+j=s+1$. That is, $H(B)$ is a time series of length $N$ reconstructed from $B$. Once this step completed, the reconstructed time series is an approximation of the original series $F$, with removed oscillating components.

\section{Study Area and Data}

In this study, monthly precipitation data of Ponel (Rezvanshahr) rain gauge station, located in Shafarud River Basin in northern Iran, were used. This station is managed and maintained by the Guilan's regional water company and the data needed to conduct this study were provided by this company. The precipitation data span the period from 1986 to 2015 (i.e. 30 years). The first 70 percent of the whole data set (i.e. the first 21 years) is used for training/calibration and the remaining 30 percent of the whole series (i.e. the remaining 9 years) is used for testing/validation. Different combinations of antecedent values of the precipitation time series were considered as input (from one to three antecedent values). The output is precipitation time series data to be forecasted in current month. Prior to use in the modelling process, all precipitation time series data is normalized into the range from 0 to 1 . 


\section{Results and Discussions}

\section{ANN Models}

As previously stated, the input combinations evaluated as input for ANN models are: (i) $P_{t-1}$, (ii) $P_{t-1}$ and $P_{t-2}$ and (iii) $P_{t-1}, P_{t-2}$ and $P_{t-3}$. In all cases, the output is the precipitation $P_{t}$ for the current month. Since recommended by Maier and Dandy (2000), the activation function used for the hidden and output layer is sigmoidal and linear type, respectively. For setting the optimal hidden neurons, a trial-and-error procedure was considered started with one hidden neuron initially, and the number of hidden neurons was progressively increased up to 10 in each trial. For each input combination, among the trained networks, to resolve the problem of overfitting, the network with the least root mean squared error (RMSE) on the validation set was selected as the optimum ANN model. Here, with increasing the number of hidden neurons, the performance of the networks in the training set generally improved while this was not true for the validation set, such that from a specific trial, the performance of the networks on the validation set had started to deteriorate, and then, among the trained networks, the last network with the best performance on the validation set was considered as the optimum network. The optimum ANN models hidden neurons for the above three input combinations were found to be 3,1 and 2 neurons respectively. The performance of the optimum ANN model for each input combination was evaluated using the root mean squared error (RMSE), correlation coefficient (R) and coefficient of efficiency (CE) criteria between the observed and forecasted values for both training and validation periods (Table 1). As suggested by Legates and McCabe (1999), a complete assessment of model performance should consider at least one absolute error measure (e.g. RMSE). $\mathrm{R}$ is an index of the degree of linear relationship between observed and forecasted data. CE indicates how well the plot of the observed values versus the forecasted values fits the 1:1 line. The table indicates that although the ANN model whose inputs are the precipitations of three previous months (input combination "iii") has the best

Table 1 RMSE, R and CE values for ANN models for both training and validation periods

\begin{tabular}{llllllll}
\hline Model inputs & \multicolumn{2}{l}{ Training } & & \multicolumn{3}{l}{ Validation } \\
\cline { 2 - 3 } & RMSE & $\mathrm{R}$ & $\mathrm{CE}$ & & RMSE & $\mathrm{R}$ & $\mathrm{CE}$ \\
\hline$P_{t-1}$ & 74.392 & 0.271 & 0.073 & & 93.000 & $\mathbf{0 . 4 5 5}$ & 0.148 \\
$P_{t-1}, P_{t-2}$ & 74.780 & 0.252 & 0.063 & & 93.515 & 0.440 & 0.139 \\
$P_{t-1}, P_{t-2}, P_{t-3}$ & $\mathbf{7 1 . 3 4 7}$ & $\mathbf{0 . 3 8 4}$ & $\mathbf{0 . 1 4 7}$ & $\mathbf{9 1 . 0 9 6}$ & 0.444 & $\mathbf{0 . 1 8 3}$
\end{tabular}

The performance criteria in boldface indicate the best ones obtained from forecasting models accuracy in both the training and validation periods; however, its $\mathrm{R}$ criterion in the validation period is slightly less than the first ANN model which is negligible. In contrast, the ANN model whose input is the precipitation of the two previous months (input combination "ii") has the worst accuracy in both the training and validation periods. The results of ANN models of input combinations of " $i$ " and "ii" indicate that here the inclusion of $P_{t-2}$ input could not able to provide useful information to the ANN model. Based on the results shown in Table 1, it should be mentioned that the performances of none of these models are satisfactory. However, among these three ANN models, the third ANN model (with input combination "iii") can be selected as the best ANN model for monthly precipitation forecasting. Figure 1 shows the hyetographs of the best ANN model between forecasted and observed precipitation series for both training and validation periods. As seen from the figure, there is no a good agreement between forecasted and observed time series which indicates that input data prepared for all the ANN models are not able to provide useful information to model the output with quality. In other words, this indicates the importance of pre-processing inputs in order to provide data with quality prior to use them in modelling, especially, using data-driven models such as ANN.

\section{ANN and SSA Conjunction Models}

As previously mentioned, raw input data preprocessing by SSA requires setting of two parameters by the user: one parameter is the window length $L$ and the other one is the number of components $r$. For the purpose of this study, the window length of 10 was found as the adequate length. The number of components can be determined based on the contribution of each component to the variance of $X$, evaluated as $\lambda_{i} / \sum_{i=1}^{L} \lambda_{i}$ : select $r$ out of $L$ components based on their contribution (Rocco 2013). In this approach, a general perception is that low contributions are associated with noise components and these noise components are usually removed from the further analysis since they are not able to provide useful information in the modelling process. Figure $2 \mathrm{a}$ illustrates the contribution of each component to the variance based on the chosen window length. As shown in the figure, the first eigenvalue accounts for $64.880 \%$ of the variance of the time series while the remaining eigenvalues account for $8.137,6.818,4.920$, $3.814,2.709,2.318,2.273,2.180$ and $1.950 \%$ respectively. However, by using this approach it is difficult to decide the optimum number of components out of the chosen window length. To overcome this situation, a separate ANN model with considering each of the above components was conducted, and the component number related to the ANN model with best performance was selected as the optimum 

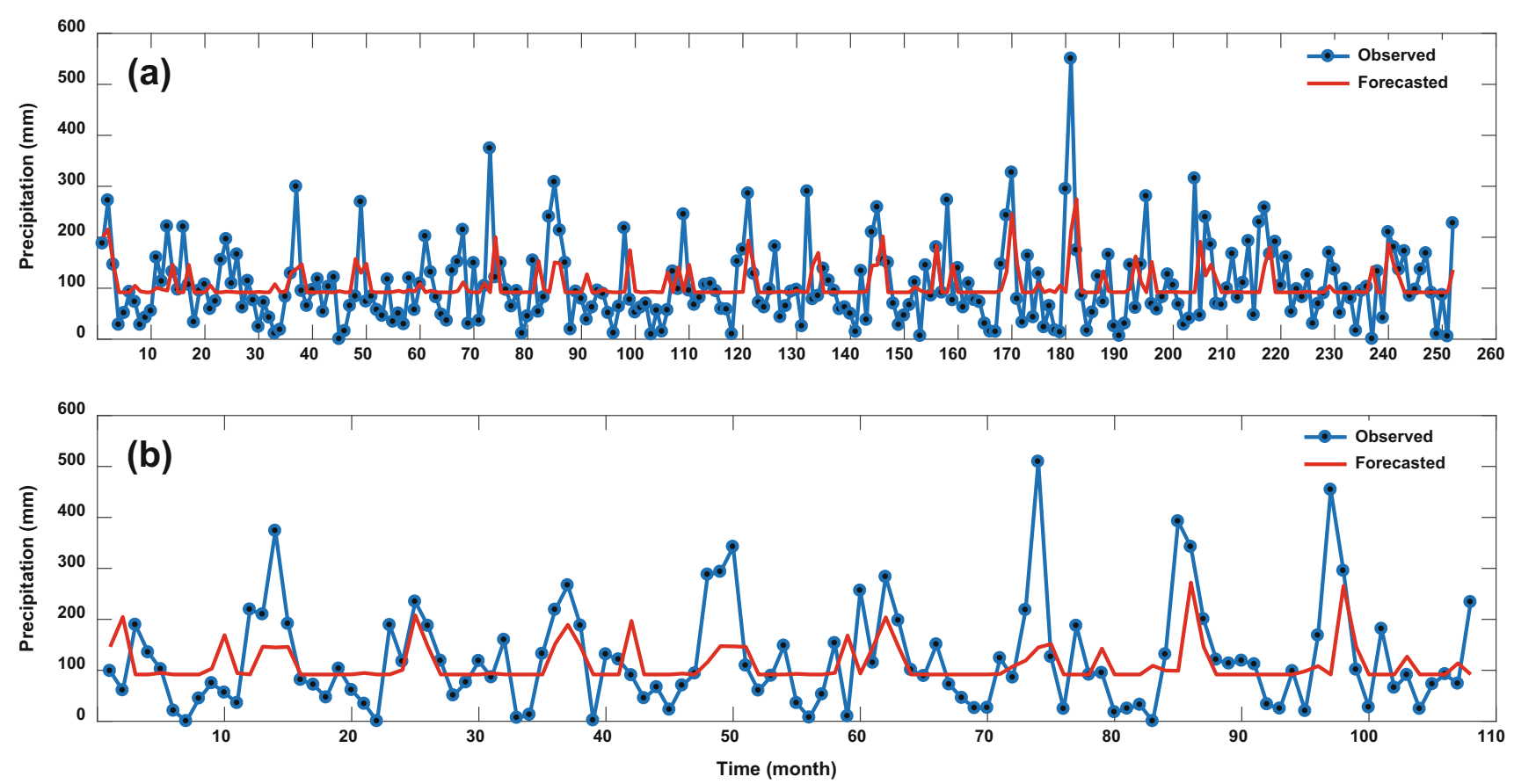

Fig. 1 The hyetographs of precipitation forecast using the best ANN model $\mathbf{a}$ for the training period and $\mathbf{b}$ for the validation period
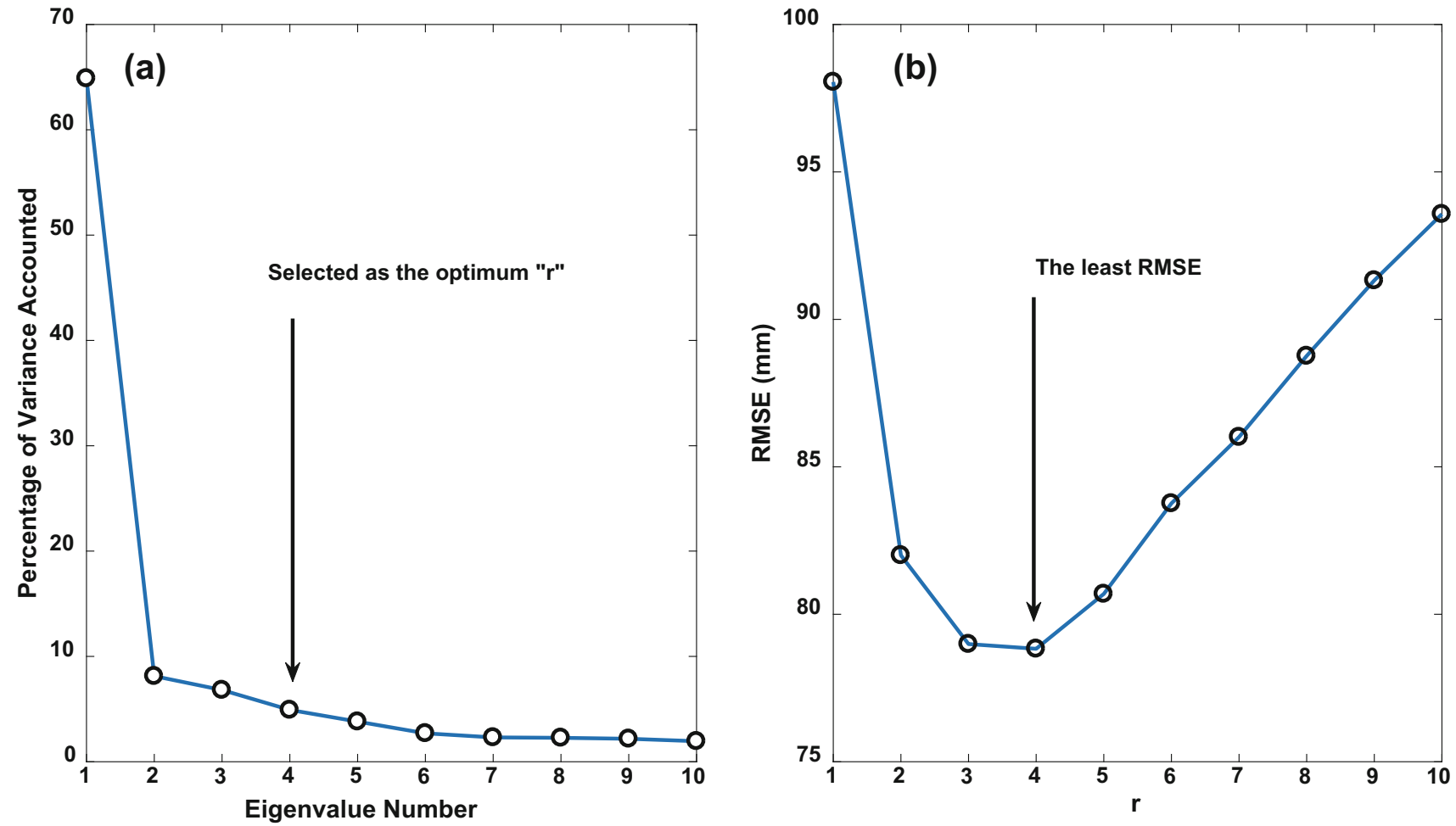

Fig. 2 a Percentage of variance accounted for each eigenvalue and $\mathbf{b}$ Performance of ANN with SSA for the first input combination in terms of RMSE as a function of $r(\leq L)$

number of components (Fig. 2b). As seen from the figure, the best performance is related to the component number of four. Hence, in this study, the first four components were selected as contributing/optimum components. Figure 3 shows original time series and reconstructed time series using SSA based on the first four components of the $P_{t-1}$. Also, this reconstructed time series must be obtained for the remaining inputs $\left(P_{t-2}\right.$ and $\left.P_{t-3}\right)$ and then they will be 


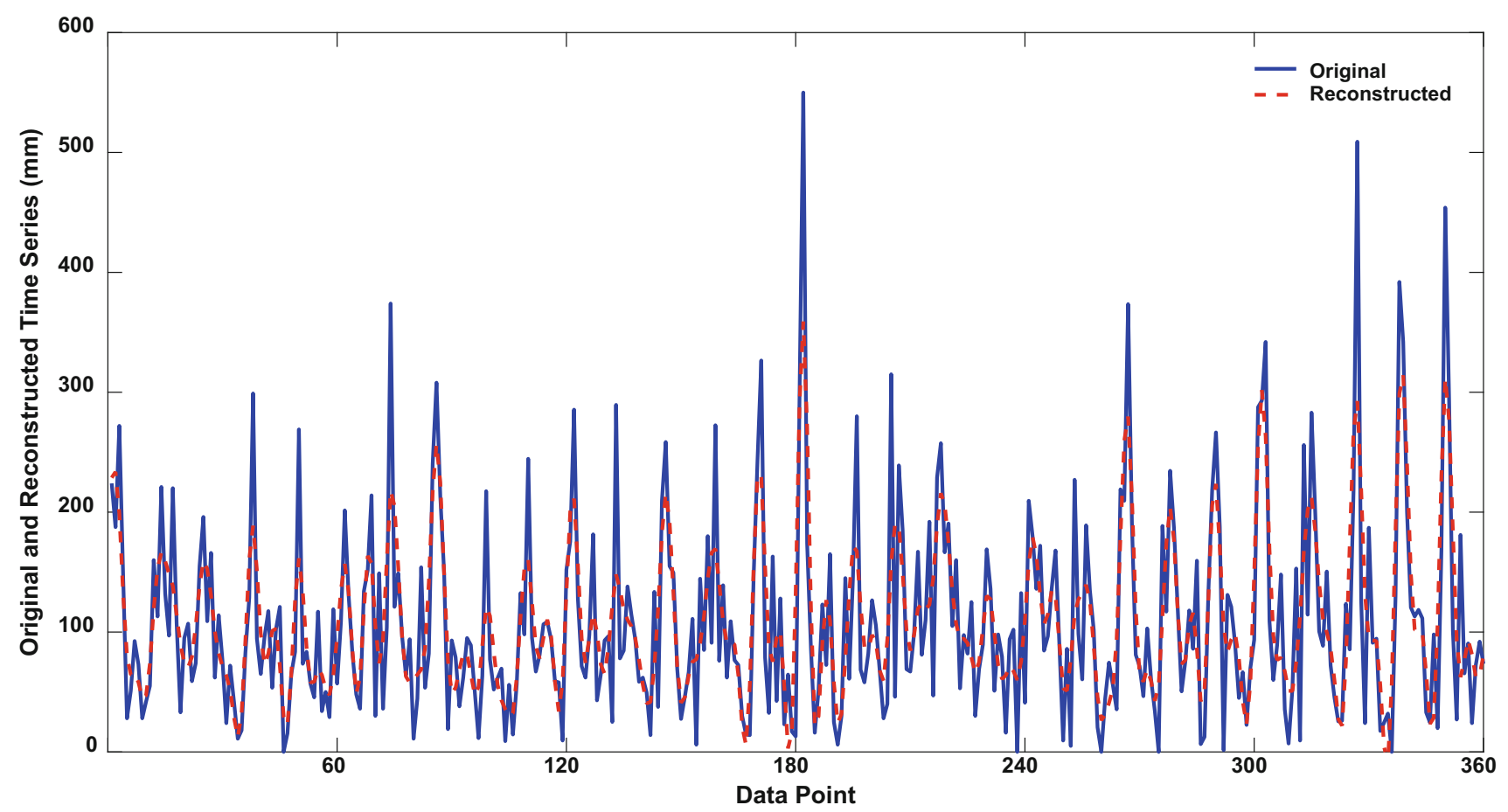

Fig. 3 Original and reconstructed time series obtained using SSA

used as input to ANN models (referred to as ANN and SSA conjunction models) instead of original input time series. The activation function used for the hidden and output layer is sigmoidal and linear type, respectively. To determine the optimal hidden neurons, a trial-and-error procedure was considered started with one hidden neuron initially, and the number of hidden neurons was progressively increased up to 10 in each trial. For each input combination reconstructed time series, the network with the least RMSE on the validation set was selected as the optimum ANN and SSA model. Therefore, the optimum ANN and SSA models hidden neurons for the above three input combinations, based on reconstructed time series using SSA as input, were found to be 1, 3 and 2 neurons respectively. RMSE, R and CE values for ANN and SSA conjunction models for both training and validation periods are shown in Table 2. It can be seen that RMSE, R and CE values of the first model for the training period are 63.278, 0.574 and 0.329 respectively and the values of these statistics for the validation set are 78.837, 0.625 and 0.388 respectively. The second model has RMSE of 50.019, R of 0.762 and $\mathrm{CE}$ of 0.581 for the training phase and RMSE of 55.879, $\mathrm{R}$ of 0.832 and $\mathrm{CE}$ of 0.692 for the validation phase. The last/third ANN and SSA conjunction model performs for the training period with RMSE, R and CE values of 49.032, 0.773 and 0.597 respectively and for the validation period with RMSE, R and CE values of 52.257, 0.858 and 0.731 respectively. According to the results, the third model is considered as the best ANN and SSA conjunction model. Figure 4 depicts the hyetographs of the best ANN and SSA conjunction model between forecasted and observed precipitation series for both training and validation periods. As evident from the figure, there is a much better agreement between forecasted and observed times series which indicates the suitability of SSA in preprocessing inputs and in providing useful information for ANN model in order to forecast the output with higher accuracy.
Table 2 RMSE, $\mathrm{R}$ and $\mathrm{CE}$ values for ANN and SSA conjunction models for both training and validation periods

\begin{tabular}{llllllll}
\hline Model inputs & \multicolumn{2}{l}{ Training } & & & \multicolumn{2}{l}{ Validation } \\
\cline { 2 - 3 } & RMSE & R & CE & & RMSE & R & CE \\
\hline$S S A\left(P_{t-1}\right)$ & 63.278 & 0.574 & 0.329 & & 78.837 & 0.625 & 0.388 \\
$S S A\left(P_{t-1}, P_{t-2}\right)$ & 50.019 & 0.762 & 0.581 & & 55.879 & 0.832 & 0.692 \\
$S S A\left(P_{t-1}, P_{t-2}, P_{t-3}\right)$ & $\mathbf{4 9 . 0 3 2}$ & $\mathbf{0 . 7 7 3}$ & $\mathbf{0 . 5 9 7}$ & & $\mathbf{5 2 . 2 5 7}$ & $\mathbf{0 . 8 5 8}$ & $\mathbf{0 . 7 3 1}$ \\
\hline
\end{tabular}

The performance criteria in boldface indicate the best ones obtained from forecasting models SSA (.) means SSA generated input time series based on the corresponding original input 

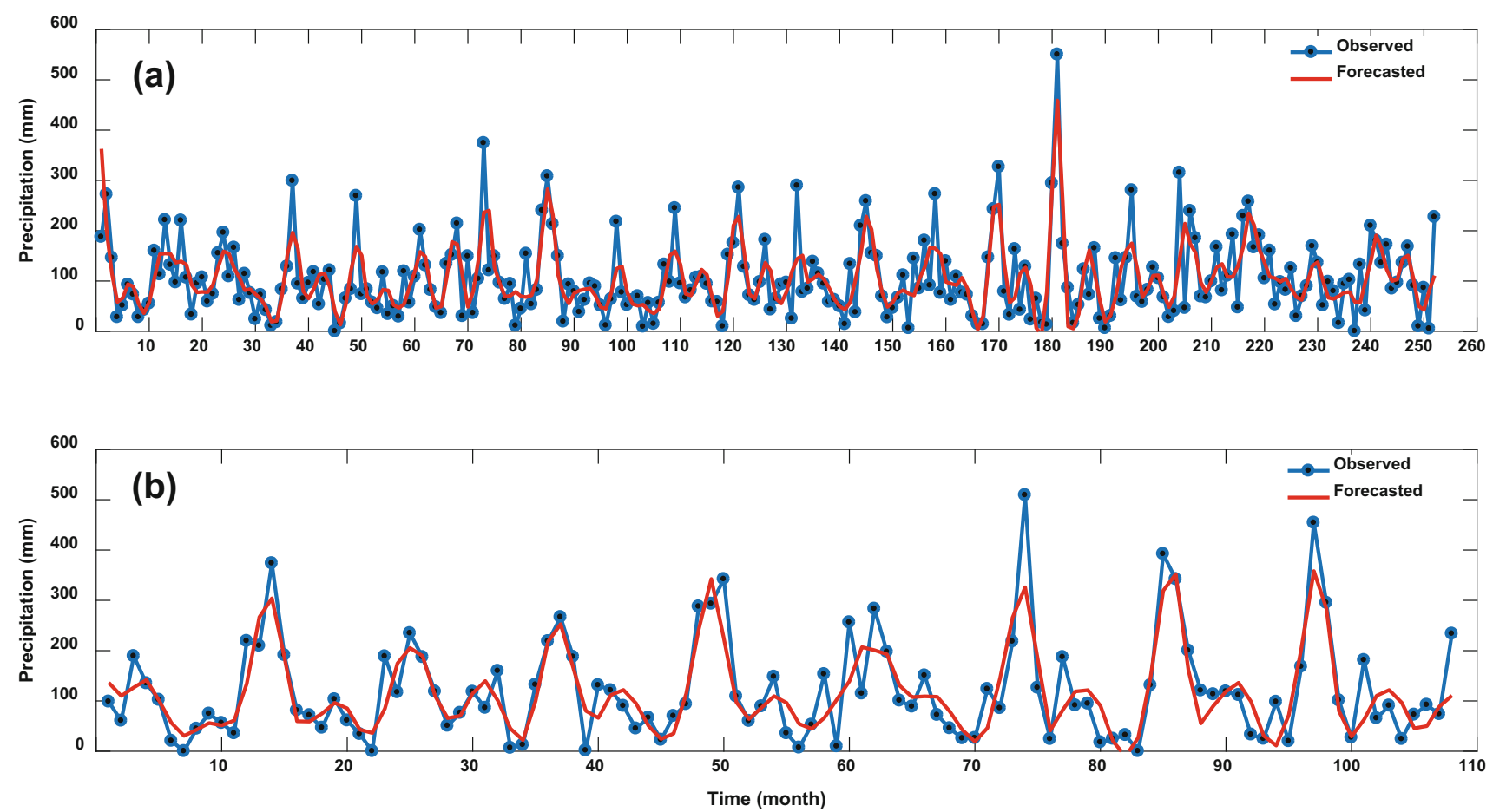

Fig. 4 The hyetographs of precipitation forecast using the best ANN and SSA conjunction model a for the training period and $\mathbf{b}$ for the validation period

\section{ANN and SSA Conjunction Models Comparison with Regular ANN Models}

It can be seen from Tables 1 and 2 that coupling/conjunction of ANN models with SSA method, in comparison with regular ANN models, could significantly improve the performance of all forecasting models. It can also be observed that although the inclusion of more inputs in regular ANN models (moving from input combination "i" to "ii") could not improve the performance; however, we could observe a slight improvement in moving from input combination "ii" to "iii" while in the case of ANN and SSA conjunction models adding more inputs to the forecasting models could improve the performance, in particular, this improvement in moving from input combination " $\mathrm{i}$ " to "ii" was significant. It is worthy to mention that both the best regular ANN and ANN with SSA models had the same optimum structure and the only difference was the inputs. These can be clearly attributed to the quality of data being used as input to the forecasting models since SSA method could provide/extract more useful information to the models from the original raw series. As previously stated, the original raw series contain some noise/random components that ultimately deteriorate the performance of the forecasting models. Also, comparing Figs. 1 and 4 indicate that the ANN and SSA conjunction model estimates the hyetograph much better than the regular ANN model. Moreover, the conjunction model could noticeably outperform the regular model in forecasting peak values.

\section{Conclusions}

This study reports a conjunction model based on artificial neural network (ANN) and singular spectrum analysis (SSA) methods for time series modelling of monthly precipitation. SSA preprocesses the input time series to provide time series without random/noise components. The conjunction model has been applied to monthly precipitation of Ponel rain gauge station in northern Iran. The input time series data of precipitation was first preprocessed by SSA and then used as input to the ANN model with original time series of the variable as output. ANN models are trained using 21 years of data and rest of the data (i.e. 9 years) is used for ANN model validation. In general, ANN and SSA conjunction model seems to be more adequate than the single ANN model for forecasting monthly precipitation. It may be noted that input data used in the ANN and SSA conjunction models were preprocessed using SSA into components without random components, which may lead to better capturing the precipitation processes. This study only used precipitation data from one rain gauge station with three previous months as inputs and further studies using more rain gauge data whose inputs are determined using more stringent approaches along with 
other input data such as temperature may be required to strengthen these findings. Moreover, the conjunction of SSA with other artificial intelligence approaches including adaptive neuro-fuzzy inference system, support vector machine and extreme learning machine may be required to compare the capability of these approaches with ANN once they are coupled with SSA.

\section{References}

Adamowski J, Sun K (2010) Development of a coupled wavelet transform and neural network method for flow forecasting of non-perennial rivers in semi-arid watersheds. $\mathrm{J}$ Hydrol 390:85-91

American Society of Civil Engineers Task Committee (2000) Artificial neural networks in hydrology-I: preliminary concepts. J Hydrol Eng 5:115-123

Chan JCL, Shi JE (1999) Prediction of the summer monsoon rainfall over South China. Int J Climatol 19(11):1255-1265

Chau KW, Wu CL (2010) A hybrid model coupled with singular spectrum analysis for daily rainfall prediction. J Hydroinform 12(4):458-473

Chen XY, Chau KW, Busari AO (2015) A comparative study of population-based optimization algorithms for downstream river flow forecasting by a hybrid neural network model. Eng Appl Artif Intel 46(A):258-268

Chu PS, He YX (1994) Long-range prediction of Hawaiian winter rainfall using canonical correlation analysis. Int $\mathrm{J}$ Climatol 14(6):659-669

DelSole T, Shukla J (2002) Linear prediction of Indian monsoon rainfall. J Clim 15(24):3645-3658

Demuth H, Beale M (1998) Neural network toolbox for use with MATLAB, users guide, version 3. The MathWorks Inc, Massachusetts

Diomede T, Davolio S, Marsigli C, Miglietta MM, Moscatello A, Papetti P, Paccagnella T, Buzzi A, Malguzzi P (2008) Discharge prediction based on multi-model precipitation forecasts. Meteorol Atmos Phys 101(3-4):245-265

El-Shafie A, Jaafer O, Seyed A (2011) Adaptive neuro-fuzzy inference system based model for rainfall forecasting in Klang River, Malaysia. Int J Phys Sci 6(12):2875-2888

Freiwan M, Cigizoglu HK (2005) Prediction of total monthly rainfall in Jordan using feed forward backpropagation method. Fresen Environ Bull 14(2):142-151

French MN, Krajewski WF, Cuykendal RR (1992) Rainfall forecasting in space and time using a neural network. J Hydrol 137:1-37

Ganguly AR, Bras RL (2003) Distributed quantitative precipitation forecasting (DQPF) using information from radar and numerical weather prediction models. J Hydrometeorol 4(6):1168-1180

Gholami V, Chau KW, Fadaee F, Torkaman J, Ghaffari A (2015) Modeling of groundwater level fluctuations using dendrochronology in alluvial aquifers. J Hydrol 529:1060-1069

Golyandina N, Korobeynikov A (2014) Basic singular spectrum analysis and forecasting with R. Comput Stat Data An 71:934-954

Golyandina N, Nekrutkin V, Zhigljavsky A (2001) Analysis of time series structure: SSA and related techniques. Chapman and Hall/ CRC

Hagan MT, Menhaj MB (1994) Training feed forward techniques with the Marquardt algorithm. IEEE $T$ Neural Netw 5(6):989-993
He S, Raghavan SV, Nguyen NS, Liong SY (2013) Ensemble rainfall forecasting with numerical weather prediction and radar-based nowcasting models. Hydrol Process 27(11):1560-1571

Kalteh AM (2013) Monthly river flow forecasting using artificial neural network and support vector regression models coupled with wavelet transform. Comput Geosci 54:1-8

Kalteh AM (2015) Wavelet genetic algorithm-support vector regression (wavelet GA-SVR) for monthly flow forecasting. Water Resour Manage 29(4):1283-1293

Kalteh AM (2016) Improving forecasting accuracy of streamflow time series using least squares support vector machine coupled with data-preprocessing techniques. Water Resour Manage 30(2):747-766

Kalteh AM, Berndtsson R (2007) Interpolating monthly precipitation by self-organizing map (SOM) and multilayer perceptron (MLP). Hydrol Sci J 52(2):305-317

Legates DR, McCabe GJ Jr (1999) Evaluating the use of goodness-offit measures in hydrologic and hydroclimatic model validation. Water Resour Res 35(1):233-241

Li F, Zeng QC (2008) Statistical prediction of East Asian summer monsoon rainfall based on SST and sea ice concentration. J Meteor Soc Jpn 86(1):237-243

Lisi F, Nicolis O, Sandri M (1995) Combining singular-spectrum analysis and neural networks for time series forecasting. Neural Process Lett 2(4):6-10

Maier HR, Dandy GC (2000) Neural networks for the prediction and forecasting of water resources variables: a review of modelling issues and applications. Environ Model Softw 15(1):101-124

Marzano FS, Fionda E, Ciotti P (2006) Neural-network approach to ground- based passive microwave estimation of precipitation intensity and extinction. J Hydrol 328:121-131

Munot AA, Kumar KK (2007) Long range prediction of Indian summer monsoon rainfall. J Earth Syst Sci 116(1):73-79

Navone HD, Ceccatto HA (1994) Predicting Indian monsoon rainfall: a neural network approach. Clim Dyn 10:305-312

Nayagam LR, Janardanan R, Mohan HSR (2008) An empirical model for the seasonal prediction of southwest monsoon rainfall over Kerala, a meteorological subdivision of India. Int J Climatol 28(6):823-831

Pongracz R, Bartholy J, Bogardi I (2001) Fuzzy rule-based prediction of monthly precipitation. Phys Chem Earth 26(9):663-667

Rocco SCM (2013) Singular spectrum analysis and forecasting of failure time series. Reliab Eng Syst Saf 114(1):126-136

Salas JD, Delleur JW, Yevjevich V, Lane WL (1985) Applied modeling of hydrologic time series. Water Resources Publications, Littleton

Sivapragasam C, Liong SY, Pasha MFK (2001) Rainfall and runoff forecasting with SSA-SVM approach. J Hydroinform 3(7):141-152

Tantanee S, Patamatammakul S, Oki T, Sriboonlue V, Prempree T (2005) Coupled wavelet-autoregressive model for annual rainfall prediction. J Environ Hydrol 13(18):1-8

Taormina R, Chau KW (2015) Data-driven input variable selection for rainfall-runoff modeling using binary-coded particle swarm optimization and Extreme Learning Machines. J Hydrol 529:1617-1632

Venkata Ramana R, Krishna B, Kumar SR, Pandey NG (2013) Monthly rainfall prediction using wavelet neural network analysis. Water Resour Manage 27:3697-3711

Wang WC, Chau KW, Xu DM, Chen XY (2015) Improving forecasting accuracy of annual runoff time series using ARIMA based on EEMD decomposition. Water Resour Manage 29(8):2655-2675 
Wu CL, Chau KW (2011) Rainfall-runoff modeling using artificial neural network coupled with singular spectrum analysis. J Hydrol 399:394-409

Wu CL, Chau KW, Fan C (2010) Prediction of rainfall time series using modular artificial neural networks coupled with datapreprocessing techniques. J Hydrol 389:146-167
Yates DN, Warner TT, Leavesley GH (2000) Prediction of a flash flood in complex terrain. Part II: A comparison of flood discharge simulations using rainfall input from radar, a dynamic model, and an automated algorithmic system. J Appl Meteorol Clim 39(6):815-825 